\title{
硼氮杂真基[4]螺烯的设计合成及性质研究*
}

\author{
段超 ${ }^{a}$ 张建伟 $b$ 向焌钧 $a$ 杨笑迪*,b高希珂*, $a$ \\ $(a$ 中国科学院大学 中国科学院上海有机化学研究所 \\ 中国科学院有机功能分子合成与组装化学重点实验室 上海 200032) \\ ( ${ }^{b}$ 上海中医药大学 创新中药研究院 上海 201203)
}

\begin{abstract}
摘要 本工作设计合成了分别含有 $\mathrm{B}-\mathrm{N}$ 键和 $\mathrm{C}=\mathrm{C}$ 键的宷基 [4]螺烯类分子 $\mathbf{1 a} / \mathbf{1 b}$ 和 $\mathbf{2}$, 其中 $\mathrm{B}-\mathrm{N}$ 键和 $\mathrm{C}=\mathrm{C}$ 键互为 等电子体. 紫外-可见吸收光谱、电化学循环伏安测试和理论计算结果表明 $\mathrm{B}-\mathrm{N}$ 键可以调控共轭骨架的电子结构及芳 香性. 单晶结构表明 $1 \mathrm{a}$ 具有螺旋几何构型, 晶体中存在 $P$ 和 $M$ 两种对映异构体. $\mathrm{B}-\mathrm{N}$ 键具有部分双键性质, 喼氮六元 环具有一定的芳香性. 大位阻基团 2,4,6-三甲基苯基(Mes)使得 $\mathbf{1 b}$ 在三氟乙酸(TFA)作用下不会发生类似于 $\mathbf{1 a}$ 的脱硼化, 而是发生和 2 相似的可逆质子响应, $\mathrm{B}-\mathrm{N}$ 键对宷单元的质子响应性质无明显影响. 三配位的喼原子可以进一步和氟离 子配位，使得 $1 \mathrm{a}$ 对氟离子有明显的选择性响应，而 $1 \mathrm{~b}$ 则因大位阻的 Mes 取代基的存在对氟离子无明显的响应性. 本 工作报道了新型宷基硼氮杂螺烯及全碳螺烯分子，为奧基多环芳烃的 “自下而上” 合成及性质研究提供了参考. 关键词 宷; 硼氮杂多环芳烃; 螺烯; 质子化
\end{abstract}

\section{Design, Synthesis and Properties of Azulene-Based BN-[4]Helicenes*}

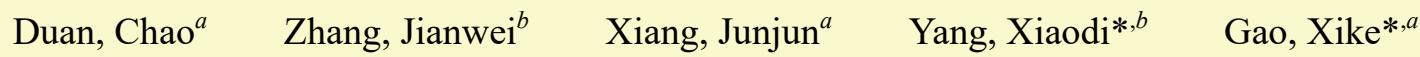 \\ ( ${ }^{a}$ Key Laboratory of Synthetic and Self-Assembly Chemistry for Organic Functional Molecules, \\ Shanghai Institute of Organic Chemistry, University of Chinese Academy of Sciences, \\ Chinese Academy of Sciences, Shanghai 200032, China) \\ ( ${ }^{b}$ Innovation Research Institute of Traditional Chinese Medicine, Shanghai University of Traditional Chinese Medicine, \\ Shanghai 201203, China)
}

\begin{abstract}
Azulene is a nonalternant and nonbenzenoid hydrocarbon with bright blue color and a dipole moment of $1.08 \mathrm{D}$, and has received increasing attention due to its unique electronic structure and physicochemical properties. Herein, we report the design and synthesis of two types of azulene-based [4] helicene $\mathbf{1 a} / \mathbf{1} \mathbf{b}$ and $\mathbf{2}$ that contain isoelectronic $\mathrm{B}-\mathrm{N}$ and $\mathrm{C}=\mathrm{C}$ units at the electron-rich 1-position of azulene unit, respectively. Formation of the helical scaffolds is executed by the introduction of boron and alkyne to flexible biaryl precursors, where the Lewis acidic boron and alkyne were employed as "glue" to join two subunits into fully fused scaffolds via electrophilic boronation and platinum-catalyzed cycloisomerization of alkyne at the 1-position of azulene unit, respectively. All of azulene-based helicenes were investigated by ultraviolet visible (UV-vis) absorption spectra, cyclic voltammetry (CV) measurements and density functional theory (DFT) calculations. Additionally, 1a was further characterized by single crystal structure analysis. The results suggest that the introduction of $\mathrm{B}-\mathrm{N}$ unit changed the electronic structure of the conjugated aromatic framework, leading to a narrow HOMO-LUMO gap. Moreover, the $\mathrm{B}-\mathrm{N}$ unit also affects the aromaticity of the $\pi$-system as revealed by nucleus-independent chemical shift (NICS) via time-dependent density functional theory (TD-DFT) calculation. The single crystal structure analysis demonstrates that 1a has a helically twisted framework and Plus $(P) / \operatorname{Minus}(M)$ enantiomers. However, the Gibbs activation energy $\left(\Delta G^{\neq}(T)\right)$ of the enantiomerization at room temperature is too low to separate two enantiomers by chiral high performance liquid chromatography (HPLC). Furthermore, the $\mathrm{B}-\mathrm{N}$ unit exhibits partial double bond character and the BN-containing six-membered ring shows weak aromaticity. 1a with a phenyl group exhibits the deboronization upon addition of trifluoroacetic acid (TFA) as well as a specific sensing behavior to fluoride ion. However, $\mathbf{1 b}$ shows no deboronization upon addition of TFA and no sensing behavior to fluoride ion due to its steric hindered mesityl (Mes) group, but has a reversible stimuli-responsiveness with acid and base, this proton-responsiveness is similar to all-carbon analogue $\mathbf{2}$.

Keywords azulene; BN-heteroaromatic; helicene; protonation
\end{abstract}

\footnotetext{
* Dedicated to the 10th anniversary of the Youth Innovation Promotion Association, CAS.

*E-mail: yangxiaodi@shutcm.edu.cn; gaoxk@mail.sioc.ac.cn

Received November 9, 2021; published December 8, 2021.

Supporting information for this article is available free of charge via the Internet at http://sioc-journal.cn.

Project supported by the National Natural Science Foundation of China (Nos. 21790362, 22075310) and the Science and Technology Commission of Shanghai Municipality (Nos. 19XD1424700, 18JC1410600).

※庆祝中国科学院青年创新促进会十年华诞.

项目受国家自然科学基金(Nos. 21790362, 22075310)和上海市科学技术委员会项目(Nos. 19XD1424700, 18JC1410600)资助.
} 


\section{1 引言}

多环芳烃(Polycyclic Aromatic Hydrocarbons, PAHs) 类化合物具有丰富的化学和分子功能, 对有机共轭材料 的发展至关重要 ${ }^{1]}$. 在 $\pi$-体系中引入如嗍、氮、硫和磷 等主族杂原子可以改变 $\pi$-电子的离域, 获得新型多环杂 芳烃(Polycyclic Heteroarenes, PHAs) 类化合物 ${ }^{[2]}$. B- N 键是 $C=C$ 键的等电子体, 其引入可以对多环芳烃骨架 的电子结构进行调控, 获得具有新性质和新功能的嗍氮 杂多环芳烃 ${ }^{[3]}$, 该类化合物目前已经应用于有机发光二

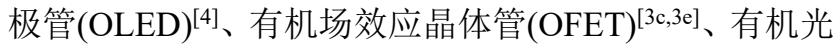
伏电池 $(\mathrm{OPV})^{[5]}$ 及指纹识别 ${ }^{[6]}$ 等领域. 螺烯(Helicene) 是 一类由芳环邻位稠合而成的非平面螺旋型多环芳烃化 合物, 具有扭曲的 $\pi$-共轭骨架及特殊的螺手性 ${ }^{[7]}$, 在手 性传感、手性光学开关、分子识别和不对称催化等领域 具有潜在的应用前景 ${ }^{[8]}$. 根据 Cahn, Ingold 和 Prelog 提 出的螺旋性规则, 螺烯的左旋和右旋对映异构体分别被 指定为 $M$ (minus) 和 $P$ (plus $)^{[9]}$, 其对映异构化能一般由 芳环的个数决定 ${ }^{[10]}$.

据我们所知，目前报道的多环(杂)芳烃和(杂)螺烯 多由苯环、噻吩、吡啶等传统芳环构成. 真(Azulene)具 有特殊的七元环并五元环芳香骨架, 其作为一种非苯类 结构砌块受到了广泛的关注. 独特的化学结构赋予姚许 多特殊的性质, 例如, 萝表现出漂亮的青蓝色, 具有约 $1.08 \mathrm{D}$ 的分子偶极矩 ${ }^{[11]}$. 宷独特的光物理性质可以通过 非镜像前线轨道分布来解释: 非镜像的最高占据分子轨 道(HOMO)和最低末占据分子轨道(LUMO)电子云分布 降低了其交叠密度, 减小了其交换积分以及未成对电子 间的排斥能, 使得 $\mathrm{S}_{0}-\mathrm{S}_{1}$ 能隙较小, 其跃迁吸收出现在可 见区 ${ }^{[12]}$. 此外, 苒的 $S_{2}-S_{1}$ 能隙较大, 导致 $S_{2} \rightarrow S_{1}$ 的非辐 射跃迁几率降低, 从而使得 $\mathrm{S}_{2} \rightarrow \mathrm{S}_{0}$ 的苂光量子产率增 大, 因此姚表现出反常的 $\mathrm{S}_{2} \rightarrow \mathrm{S}_{0}$ 苂光现象, 也被称为 “反 Kasha 规则 $\left(\mathrm{S}_{1} \rightarrow \mathrm{S}_{0}\right)$ ” 苂光 ${ }^{[13]}$. 菜独特的化学结构和 物理化学性质使其成为构筑具有特殊拓扑结构的多环 (杂)芳烃和(杂)螺烯的理想砌块 ${ }^{[14]}$.

然而, 宷基多环(杂)芳烃特别是奧基(杂)螺烯分子 的合成及衍生化通常较为困难, 且大都是采用“后合成” (late synthetic stage) 策略获得 ${ }^{[15]}$, 存在一定的随机性和 偶然性, 这在一定程度上制约了宷基多环(杂)芳烃和 (杂)螺烯的功能化应用. 因此, 发展 “自下而上” 的精准 合成策略对于发展萝基多环(杂)芳烃和(杂)螺烯及其功 能化应用具有重要的科学意义. 据我们了解, 目前只有 几例真基螺烯报道, 且几乎都是通过 “后合成” 策略获 得 ${ }^{[16]}$. 基于此, 我们利用 “自下而上” 的合成策略设计 合成了两例具有不同位阻取代基的硼氮杂奧基 [4]螺烯 分子 $1 \mathrm{a}$ 和 $1 \mathrm{~b}$. 作为对比, 我们进一步合成了具有等电 子骨架的全碳螺烯分子 2. 我们通过实验分析和理论计 算对三个螺烯分子的基本物理化学性质进行了详细研 究, 并研究了 $\mathrm{B}-\mathrm{N}$ 键对宷基螺烯分子性质的影响.

\section{2 结果与讨论}

\section{1 合成与结构表征}

$1 \mathrm{a} / \mathbf{1 b}$ 和 2 的合成路线如图式 1 所示, 我们参照文 献方法合成了化合物 $\mathbf{S} 2^{[17]}$ 和 $\mathbf{S 6} 6^{[18]}$. S2 和 $\mathbf{S} 3^{[14 a]}$ 经过 Suzuki-Miyaura 偶联反应以 $61 \%$ 的产率得到中间体 S4. S4 和亲电试剂二氯苯硼烷 $\left(\mathrm{PhBCl}_{2}\right)$ 进行亲电硼化反应 以 79\%的产率得到了苯基取代的螺烯 1a. 我们将亲电 试剂替换为三氯化硼 $\left(\mathrm{BCl}_{3}\right)$ 得到了一个氯代中间体, 随 后利用 2,4,6-三甲基苯基溴化镁( $\mathrm{MesMgBr}$ )进行原位亲 核取代得到了 Mes 基团取代的 $\mathbf{1 b}$ ，总产率为 $75 \%$. 中间 体 S6 和 1-辛炔通过 Sonogashira 偶联反应以 $97 \%$ 的产率 得到了中间体 S7, S7 与 S3 发生 Suzuki-Miyaura 偶联反 应以 $87 \%$ 的产率得到了关键中间体 S8. 随后 $\mathbf{S 8}$ 在二氯 化铂 $\left(\mathrm{PtCl}_{2}\right)$ 催化下发生环化异构化反应以 $63 \%$ 的产率得 到螺烯 2. 三个螺烯分子在常规有机溶剂中均具有良好 的溶解性，我们利用核磁共振氢谱 $\left.{ }^{1} \mathrm{H} N \mathrm{NM}\right)$ 、核磁共振 碳谱 $\left({ }^{13} \mathrm{C} \mathrm{NMR}\right)$ 和高分辨质谱(HRMS)等手段对螺烯 $\mathbf{1 a} / \mathbf{1 b}$ 和 2 的结构进行了表征.
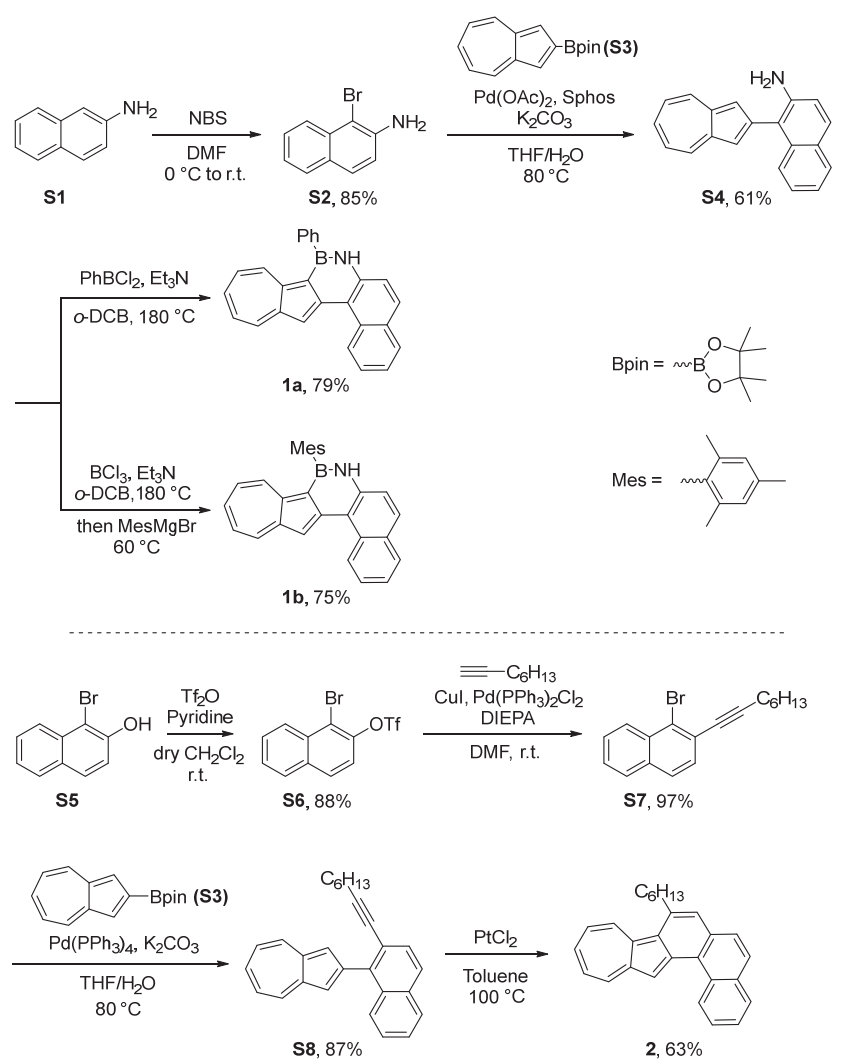

图式 1 真基螺烯 $1 a / 1 b$ 和 2 的合成路线

Scheme 1 Synthesis routes of azulene-embedded helicenes $1 \mathbf{a} / \mathbf{1} \mathbf{b}$ and $\mathbf{2}$

如图 1 所示, $1 \mathbf{a}, \mathbf{1 b}$ 和 2 中 $\mathrm{H}-1$ 的化学位移分别位 于 $\delta 8.70,8.75$ 和 $8.75, \mathrm{H}-2$ 的化学位移分别位于 $\delta 9.41$, 9.45 和 9.45, 其相对于 $\mathbf{S} 4$ 中的 H-1 ( $\delta$ 7.55)和 H-2 ( $\delta$ 7.74) 明显向低场移动, 原因是螺旋结构形成了湾区 
(cove-region) ${ }^{[19]}$, 该变化趋势和 [4]螺烯一致 ${ }^{[20]}$. 另一方 面, $1 \mathbf{b}$ 中的 H-3 ( $\delta$ 8.38) 与 $\mathbf{1 a}(\delta 8.92)$ 和 $\mathbf{2}(\delta$ 8.87)中的 H-3 相比受到的屏蔽效应增大, 化学位移移向高场. 此 外, $1 \mathrm{a}$ 和 $1 \mathrm{~b}$ 的氮原子上的 $\mathrm{H}$ 化学位移出现在芳香区 $(\delta$ 7.98), 表明嗍氮六元环具有一定的芳香性.

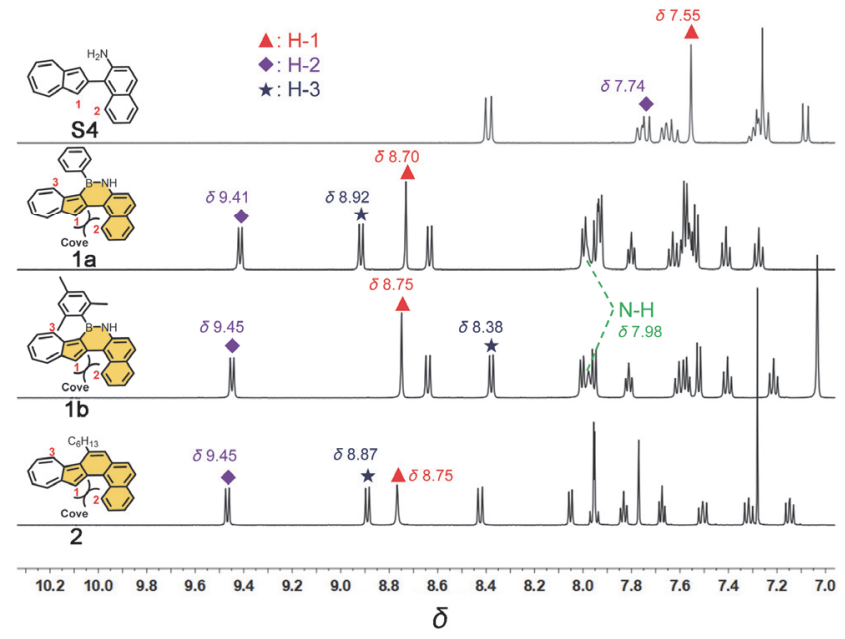

图 1 化合物 $\mathbf{S 4}, \mathbf{1 a} / \mathbf{1 b}$ 及 2 的部分 ${ }^{1} \mathrm{H} N M R$ 谱图

Figure 1 Partial ${ }^{1} \mathrm{H}$ NMR spectra of compounds $\mathrm{S4}, \mathbf{1 a} / \mathbf{1 b}$ and $\mathbf{2}$

\section{2 单晶 $X$ 射线衍射及理论计算}

为进一步研究分子的精确结构和堆积方式, 通过乙 腈和正己烷混合溶剂缓慢挥发获得 1a 的单晶, 并对其 进行 $X$ 射线单晶衍射测试与结构解析 (1b 和 2 的单晶结 构未能获得). $1 \mathrm{a}$ 的单晶结构如图 $2 \mathrm{a}$ 所示(为方便观察绘 图时已略去氢原子). 1a 中的 $\mathrm{B}(2)-\mathrm{N}(1)$ 键长 $(0.1418 \mathrm{~nm})$ 较之经典的 $\mathrm{B}-\mathrm{N}$ 键长 $(0.1478 \sim 0.1488 \mathrm{~nm})^{[21]}$ 更短, 和

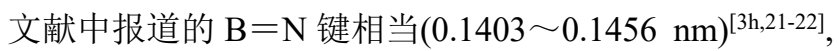
表明 $\mathrm{B}(2)-\mathrm{N}(1)$ 键具有部分双键性质. 此外, 环内的

(a)
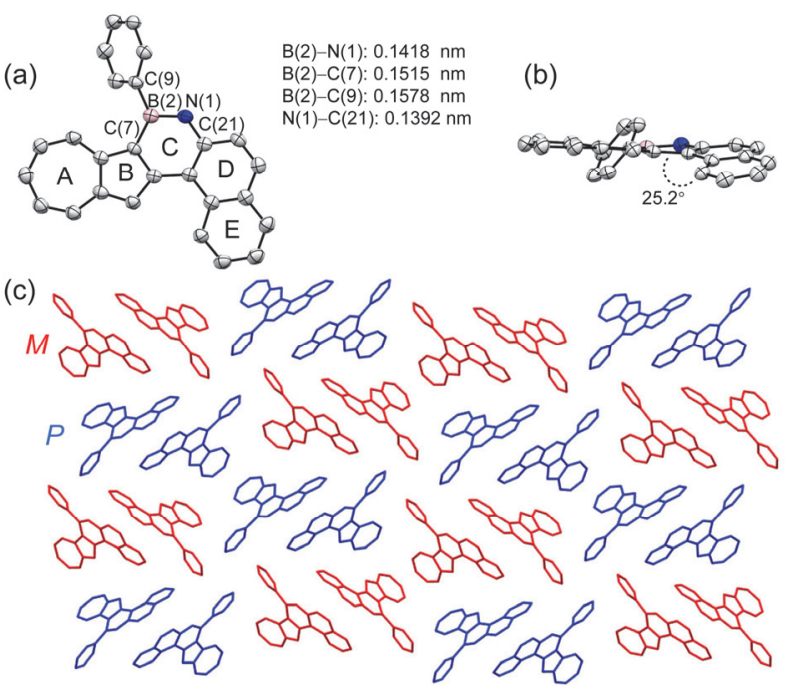

图 $21 \mathrm{a}$ 的单晶结构(已略去氢原子): (a) 俯视图, (b) 侧视图, (c) 堆 积图(红色: $M$ 对映异构体; 蓝色: $P$ 对映异构体)

Figure 2 Crystal structure of 1a (hydrogen atoms are omitted): (a) top view, (b) side view, and (c) packing diagrams (red: $M$ enantiomer; blue: $P$ enantiomer)
$\mathrm{B}(2)-\mathrm{C}(7)$ 键长为 $0.1515 \mathrm{~nm}$, 比环外的 $\mathrm{B}(2)-\mathrm{C}(9)$ $(0.1578 \mathrm{~nm})$ 的键长更短, 且 $\mathrm{N}(1)-\mathrm{C}(21)$ 的键长 $(0.1392$ $\mathrm{nm})$ 也比典型的 $\mathrm{N}-\mathrm{C}$ 键 $(\approx 0.148 \mathrm{~nm})^{[21]}$ 更短, 表明喼氮 六元杂环 $\mathrm{C}$ 的键长趋于平均化，具有一定的芳香性，这 与 ${ }^{1} \mathrm{H}$ NMR 分析结果一致. 螺烯骨架中的三个芳环 $(B, C$ 和 D)均发生一定程度的扭曲(图 2b), 宷的五元环 B 和末 端苯环 $\mathrm{E}$ 之间的二面角为 $25.2^{\circ}$, 与 [4] 螺烯相近 ${ }^{[23]}$, 表 明 $1 \mathrm{a}$ 具有典型的螺烯结构. 另一方面, 硼原子上取代的 苯环和嘲氮六元杂环 $\mathrm{C}$ 的二面角为 $62.4^{\circ}$, 阻碍了苯环 与螺烯骨架之间的共轭. 此外, $1 \mathrm{a}$ 单晶结构中存在 $P$ 和 $M$ 两种对映异构体(图 2c), 两种对映异构体分别形成二 聚体，在晶体中交替形成柱状堆积. 由于[4]螺烯的对映 异构化能垒较低 ${ }^{[24]}$, 两种对映异构体未能成功分离. 此 外，扭曲的螺烯构型及嗍原子上的苯基明显阻碍了分子 间 $\pi-\pi$ 相互作用.

通过 Gaussian16 在 DFT/B3LYP/6-311G(d,p)计算水 平上对 $1 \mathrm{a}$ 和 2 的分子结构进行优化，并对其分子前线轨 道的电子密度分布与轨道能级进行了对比分析(1a 和 $\mathbf{1 b}$ 骨架相同，因此未对 $1 \mathrm{~b}$ 进行计算). 图 3 显示了 $1 \mathbf{a}$ 和 2 的前线轨道能级和轨道分布情况. $1 \mathbf{a}$ 和 2 的 HOMO 轨道 电子云离域在整个共轭分子骨架上，计算得到两者的 $\mathrm{HOMO}$ 能级分别为 $-5.36 \mathrm{eV}$ 和 $-5.13 \mathrm{eV}$. 另一方面, $1 \mathrm{a}$ 的 LUMO 轨道电子云主要集中在宷单元及其相连䓺基 的内侧苯环上, $\mathrm{B}-\mathrm{N}$ 键和菜基外侧苯环上电子云分布 较少，原因可能是宷的 LUMO 电子云密度主要集中在 奧的偶数位 ${ }^{[25]}$, 使奇数位点难以与 $\mathrm{B}-\mathrm{N}$ 键形成良好的 离域. 2 的 LUMO 轨道电子云分布情况与 $\mathbf{1 a}$ 相似, $\mathrm{C}=\mathrm{C}$ 键 $(2$ 中红色部分, $\mathrm{B}-\mathrm{N}$ 键的等电子体)仍然分布较少.

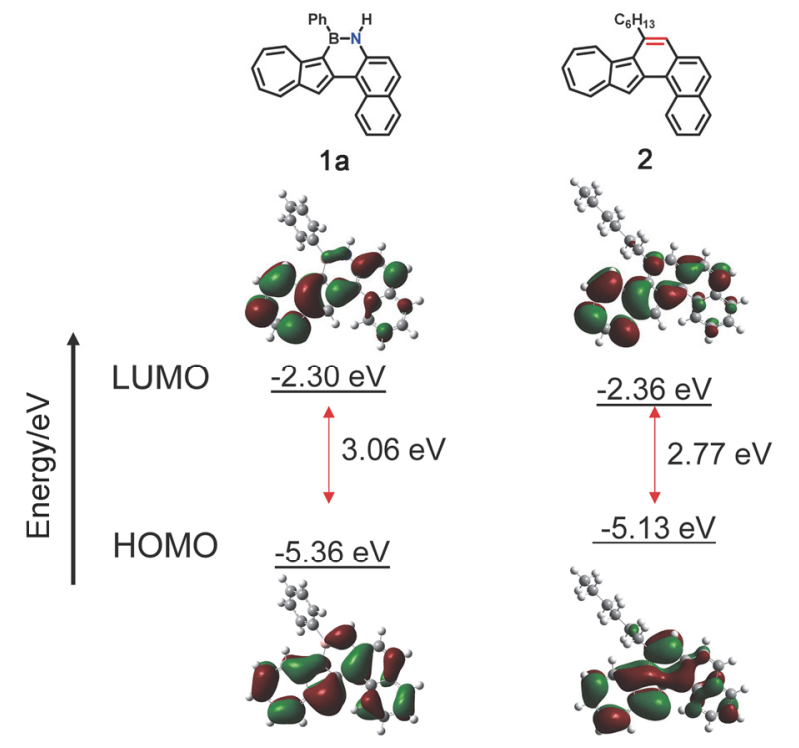

图 3 DFT 计算获得的 $1 \mathbf{a}$ 和 $\mathbf{2}$ 的前线轨道及轨道能级

Figure 3 Frontier molecular orbitals and energy levels of 1a and 2 obtained by DFT calculations 
计算得到 $1 \mathrm{a}$ 和 2 的 LUMO 能级分别为 $-2.30 \mathrm{eV}$ 和 $-2.36 \mathrm{eV}$, HOMO-LUMO 能隙分别为 $3.06 \mathrm{eV}$ 和 2.77 $\mathrm{eV}$, 前者相对于后者有所拓宽, 原因是奧的 1-位上强吸 电子性的硼原子有效降低了分子 $\mathrm{HOMO}$ 能级, 但对

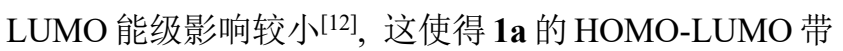
隙变宽 $0.29 \mathrm{eV}$, 表明 $\mathrm{B}-\mathrm{N}$ 键替换 $\mathrm{C}=\mathrm{C}$ 键后改变了骨 架的电子结构, 具备有效调控材料光物理性质的可行性.

单晶结构表明, B- $\mathrm{N}$ 键具有一定的双键性质, 六元 嗍氮杂环具有芳香性特征. 我们通过计算核独立化学位 移(NICS)进一步评价了 $\mathrm{B}-\mathrm{N}$ 键对骨架芳香性的影响 (图 4). 结果表明: 相比于 $\mathbf{2}$ 中的六元环 $C^{\prime}, \mathbf{1 a}$ 的硼氮杂 环 C 的芳香性更弱(NICS (1): - 3.97 vs. -9.73), 这与 ${ }^{1} \mathrm{H}$ NMR 及单晶数据分析结果一致. 此外, 苒和䒺环上 的 NICS (1)值的改变表明 B-N 键对螺烯骨架的电子结 构产生了影响, 可能是嗍原子较强的缺电性影响了电子 在 $\pi$-骨架上的离域.
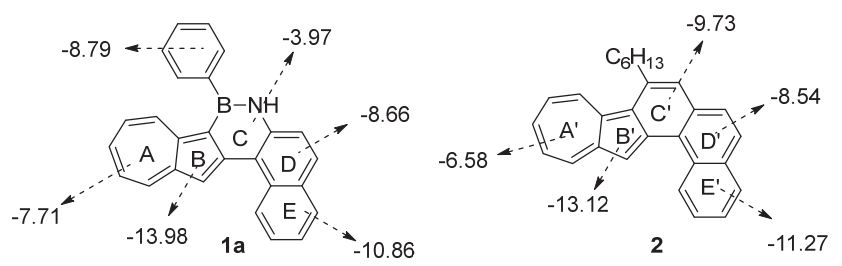

图 $41 \mathrm{a}$ 和 2 的 NICS (1)值

Figure 4 NICS (1) values of $\mathbf{1 a}$ and $\mathbf{2}$

\section{3 光谱和电化学性质}

图 $5 \mathrm{a}$ 给出了 $1 \mathrm{a} / 1 \mathrm{~b}$ 和 2 在二氯甲烷溶液中的紫外可见吸收光谱. $1 \mathrm{a}$ 和 $1 \mathrm{~b}$ 的吸收光谱几乎重叠, 最大吸收 波长为 $305 \mathrm{~nm}\left(\varepsilon=31000 \mathrm{~L} \cdot \mathrm{mol}^{-1} \cdot \mathrm{cm}^{-1}\right)$ 和 $332 \mathrm{~nm}(\varepsilon=$ $\left.33000 \mathrm{~L} \cdot \mathrm{mol}^{-1} \cdot \mathrm{cm}^{-1}\right)$; 此外, 两者在 $370 \mathrm{~nm}(\varepsilon=16000$ $\mathrm{L} \cdot \mathrm{mol}^{-1} \cdot \mathrm{cm}^{-1}$ ) 附近有一个肩峰, 这是因为两者的共轭骨 架相同，仅嗍原子上的芳基取代基不同，且芳基取代基 与骨架之间的共轭被阻断. 2 的最大吸收波长为 $350 \mathrm{~nm}$ $\left(\varepsilon=58000 \mathrm{~L} \cdot \mathrm{mol}^{-1} \cdot \mathrm{cm}^{-1}\right)$, 相对于 $1 \mathbf{a}$ 和 $\mathbf{1 b}$ 红移了约 18

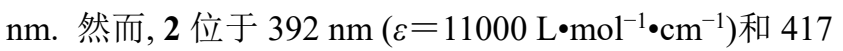

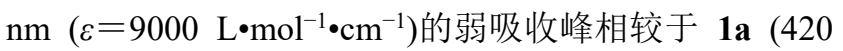
$\mathrm{nm}, \varepsilon=6400 \mathrm{~L} \cdot \mathrm{mol}^{-1} \cdot \mathrm{cm}^{-1}$ 和 $444 \mathrm{~nm}, \varepsilon=6800$ $\left.\mathrm{L} \cdot \mathrm{mol}^{-1} \cdot \mathrm{cm}^{-1}\right)$ 和 $\mathbf{1 b}\left(419 \mathrm{~nm}, \varepsilon=7300 \mathrm{~L} \cdot \mathrm{mol}^{-1} \cdot \mathrm{cm}^{-1}\right.$ 和 $\left.442 \mathrm{~nm}, \varepsilon=7900 \mathrm{~L} \cdot \mathrm{mol}^{-1} \cdot \mathrm{cm}^{-1}\right)$ 有一定程度的蓝移, 表明 $\mathrm{B}-\mathrm{N}$ 键改变了共轭骨架的 $\pi$-电子结构, 导致 $\mathbf{~ a / 1 b}$ 和 2 具有明显不同的吸收谱带. $1 \mathrm{a}$ 和 2 的吸收光谱与含时密 度泛函理论(TD-DFT)计算模拟得到的光谱曲线相一致. 根据 TD-DFT 计算结果显示, 1a 在 500 600 nm 之间的 弱吸收峰 $(f=0.0190)$ 可归结于 HOMO-1-LUMO (20\%)和 HOMO-LUMO (77\%)的跃迁, 2 在 $600 \mathrm{~nm}$ 附近的弱吸收 峰 $(f=0.0098)$ 是由 HOMO-LUMO $(97 \%)$ 跃迁引起的(见 支持信息, 图 S1), 这是宷的特征吸收峰, 与其他宷类衍 生物相似 ${ }^{[14 a, 14 c, 26]}$. 硼氮杂多环芳烃通常具有优良的荧 光性质 ${ }^{[27]}$. 我们在二氯甲烷溶液中测得了 $\mathbf{1 a} / \mathbf{1 b}$ 和 $\mathbf{2}$ 的
发射光谱(见支持信息, 图 S2), 三个螺烯分子均表现出 微弱的菼光，三者的最大发射波长均为 $384 \mathrm{~nm}$ ，量子产 率分别为 $0.15 \%, 0.08 \%$ 和 $0.12 \%$. 较弱的发射峰可归结 于 $\mathrm{S}_{2} \rightarrow \mathrm{S}_{0}$ 的辐射跃迁，即 “反 Kasha 规则” 苂光，这与 其他宷类衍生物类似 ${ }^{[14 \mathrm{a}, 14 \mathrm{c}, 28]}$.

我们利用循环伏安法 $(\mathrm{CV})$ 测试了 $\mathbf{1 a} / \mathbf{1 b}$ 和 2 的电化 学性质, 三者均在正向出现不可逆的氧化峰, 表明 $1 \mathrm{a} / \mathbf{1 b}$ 和 2 在溶液中存在不可逆的氧化过程(图 $5 \mathrm{~b}$ ). 由切 线法得到 1a, $1 \mathbf{b}$ 和 2 的起始氧化电位 $E_{\mathrm{ox}}^{\mathrm{onset}}$ 分别为 0.41 $\mathrm{V}, 0.46 \mathrm{~V}$ 和 $0.16 \mathrm{~V}$ (相对于二茂铁, 相同条件下测得二 茂铁 $\left(\mathrm{Fc} / \mathrm{Fc}^{+}\right)$相对于甘录电极的电位为 $0.48 \mathrm{~V}$, 二茂铁的

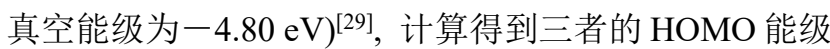
分别为 $-5.21 \mathrm{eV},-5.26 \mathrm{eV}$ 和 $-4.96 \mathrm{eV}$ ，表明 2 相对于 $\mathbf{1 a} / \mathbf{1 b}$ 具有更强的给电子能力. 另一方面, 三个化合物 都在负向出现一组准可逆的氧化还原峰, 其起始还原电 位 $E_{\mathrm{red}}^{\mathrm{onset}}$ 分别为 $-1.89 \mathrm{~V},-1.93 \mathrm{~V}$ 和 $-1.85 \mathrm{~V}$ (相对于二 茂铁), 三者相差较小, 表明它们具有相似的还原稳定 性, 计算得到 $1 \mathbf{a}, \mathbf{1 b}$ 和 $\mathbf{2}$ 的 LUMO 能级分别为 $-2.91 \mathrm{eV}$, $-2.87 \mathrm{eV}$ 和 $-2.95 \mathrm{eV}$. 如表 1 所示, 由 $\mathrm{CV}$ 测得的前线 轨道能级和 DFT 计算结果相差较小.
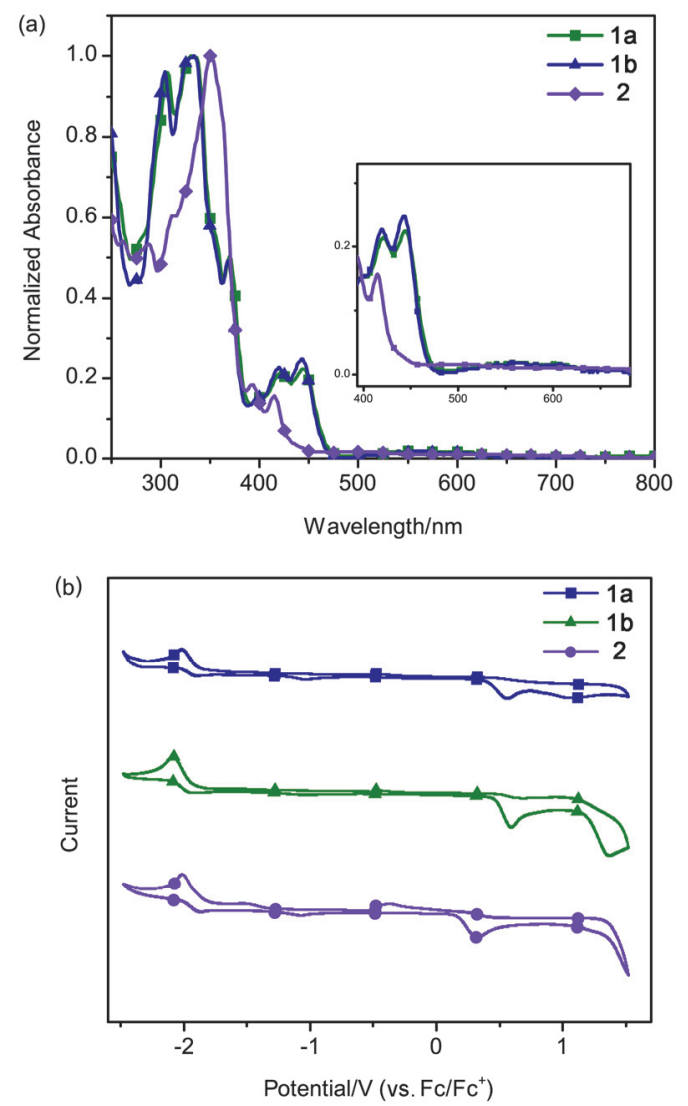

图 5 (a) $1 \mathbf{a} / \mathbf{1 b}$ 和 2 在二氯甲烷溶液中的紫外-可见吸收光谱 $\left(1 \times 10^{-5}\right.$ $\mathrm{mol} / \mathrm{L}) ;\left(\right.$ b) $\mathbf{1 a} / \mathbf{1 b}$ 和 2 在二氯甲烷 $\left(0.1 \mathrm{~mol} / \mathrm{L} \mathrm{Bu}_{4} \mathrm{NPF}_{6}\right.$ 作为支持电解质, 甘录电极作为参比, 扫描速率 $100 \mathrm{mV} / \mathrm{s}$ )中的循环伏安曲线

Figure 5 (a) UV-Vis absorption spectra of $\mathbf{1 a} / \mathbf{1 b}$ and $\mathbf{2}$ in dichloromethane solutions $\left(1 \times 10^{-5} \mathrm{~mol} / \mathrm{L}\right)$; (b) cyclic voltammograms of $\mathbf{1 a} / \mathbf{1} \mathbf{b}$ and $\mathbf{2 a}$ in dichloromethane $\left(0.1 \mathrm{~mol} / \mathrm{L} \mathrm{Bu}_{4} \mathrm{NPF}_{6}\right.$ as supporting electrolyte, $\mathrm{SCE}$ as reference electrode, scan rate of $100 \mathrm{mV} / \mathrm{s}$ ) 
表 $1 \quad 1 \mathrm{a} / \mathbf{1 b}$ 和 2 的光谱、电化学和理论计算数据

Table 1 Optical, electrochemical and DFT calculations data for $\mathbf{1 a} / \mathbf{1} \mathbf{b}$ and $\mathbf{2}$

\begin{tabular}{cccccccc}
\hline Compound & $\lambda_{\max } / \mathrm{nm}$ & $E_{\mathrm{g}}^{\text {opt } a} / \mathrm{V}$ & $E_{\mathrm{HOMO}} / \mathrm{eV}$ & $E_{\mathrm{LUMO}}{ }^{b} / \mathrm{eV}$ & $E_{\mathrm{HOMO}} c / \mathrm{eV}$ & $E_{\mathrm{LUMO}} / \mathrm{eV}$ & $E_{\mathrm{g}}{ }^{c} / \mathrm{V}$ \\
\hline $\mathbf{1 a}$ & 305,332 & 2.66 & -5.21 & -2.91 & -5.36 & -2.30 & 3.06 \\
$\mathbf{1 b}$ & 305,332 & 2.67 & -5.26 & -2.87 & - & - & - \\
$\mathbf{2}$ & 350 & 2.72 & -4.96 & -2.95 & -5.13 & -2.36 & 2.77 \\
\hline
\end{tabular}

${ }^{a}$ Estimated from the onset absorption $\lambda_{\mathrm{onset}}{ }^{b}$ calculated from $E_{\mathrm{HOMO}}=-4.80-E_{\mathrm{ox}}^{\text {onset }} / E_{\mathrm{red}}^{\text {onset }}$ (calibration by ferrocene); ${ }^{c}$ estimated from DFT calculations.

\section{4 酸刺激响应性质研究}

宷及其衍生物对酸、碱具有一定的化学敏感性，可 以在强酸如三氟乙酸(TFA)、三氟甲磺酸(TfOH)作用下 发生质子化使七元环形成稳定的卓鎓离子[26a,30]. 由于 1a 在 TFA 作用下会发生脱硼化反应(见支持信息, 图式

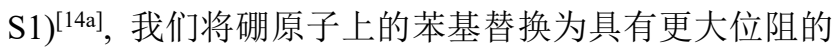
Mes 基以保护硼原子. 如图 6 所示, 加入 TFA 后, 1b 在 $300 \sim 450 \mathrm{~nm}$ 区间的吸收峰逐渐减弱, 同时在约 $515 \mathrm{~nm}$ 处出现一个宽的吸收峰, 加入过量三乙胺后, 吸收峰可 恢复(图 6a), 表明 1b 在加入 TFA 后没有发生脱硼化, 而 是发生了可逆的质子化与去质子化过程. 2 在 TFA 作用 下也发生与 $1 \mathrm{~b}$ 相似的可逆质子化过程(图 6b), 二者均 在 $n_{\mathrm{TFA}} / n_{\mathbf{x}}=5(\mathrm{X}=\mathbf{1 b}$ 和 2$)$ 时达到饱和, 表明 $\mathrm{B}-\mathrm{N}$ 键不 会影响宷单元的质子化响应.
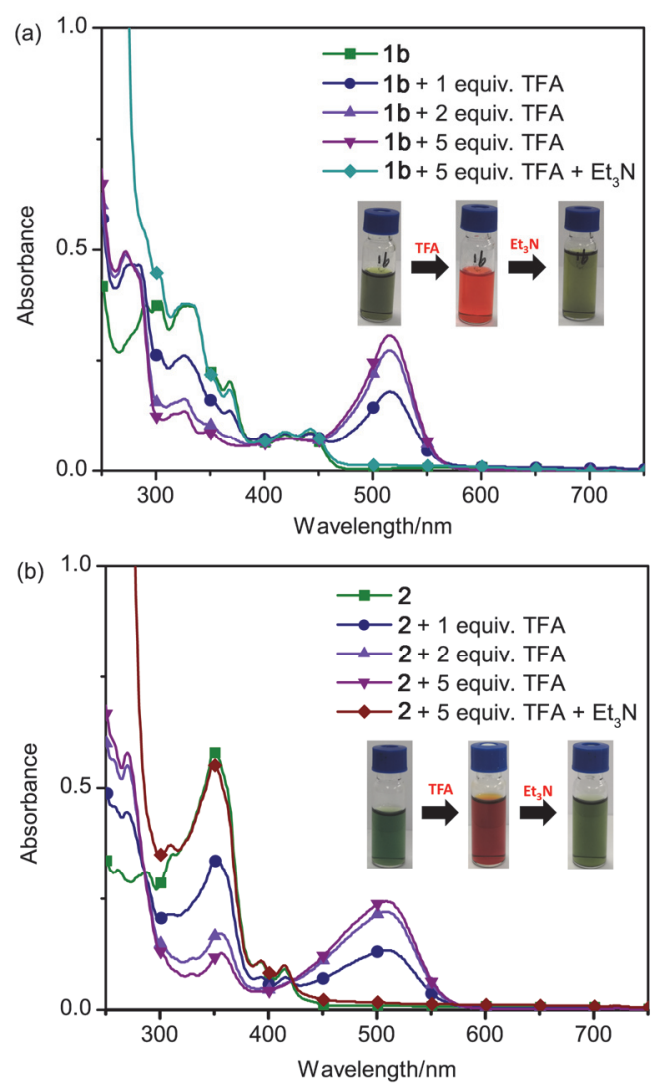

图 6 (a) $1 \mathrm{~b}$ 在二氯甲烷溶液中质子化和去质子化过程的紫外-可见吸 收光谱及颜色变化; (b) 2 在二氯甲烷溶液中质子化和去质子化过程的 紫外-可见吸收光谱及颜色变化

Figure 6 (a) UV-Vis absorption spectra and colour change of $\mathbf{1 b}$ during protonation and deprotonation in dichloromethane solutions; (b) UV-Vis absorption spectra and colour change of $\mathbf{2}$ during protonation and deprotonation in dichloromethane solutions

\section{5 氟离子响应性质研究}

三配位的硼原子存在一个空的 $\mathrm{p}$ 轨道, 可以接受一 对孤对电子的进攻变成四配位，进而调控有机硼化合物 的光电性质 $[22 \mathrm{c}, 31]$, 基于此机理可发展有机硼类氟离子 光学检测材料 ${ }^{[32]}$. 我们利用紫外-可见吸收光谱研究了 $1 \mathrm{a}$ 和 $1 \mathrm{~b}$ 对氟离子的响应性质, 如图 7a 所示, $1 \mathrm{a}$ 的二氯 甲烷溶液中加入不同阴离子的四丁基铵盐 TBAX $\left(n_{\mathrm{TBAX}} / n_{1 \mathrm{a}}=10, \mathrm{X}\right.$ 为不同阴离子 $)$, 仅加入 $\mathrm{TBAF}$ 后在 $525 \mathrm{~nm}$ 处出现一个宽的吸收峰, 溶液颜色由绿色变为 紫色，表明共轭骨架的电子跃迁模式发生了改变. 加入 其他阴离子后其吸收光谱保持不变, 表明 1a 对氟离子
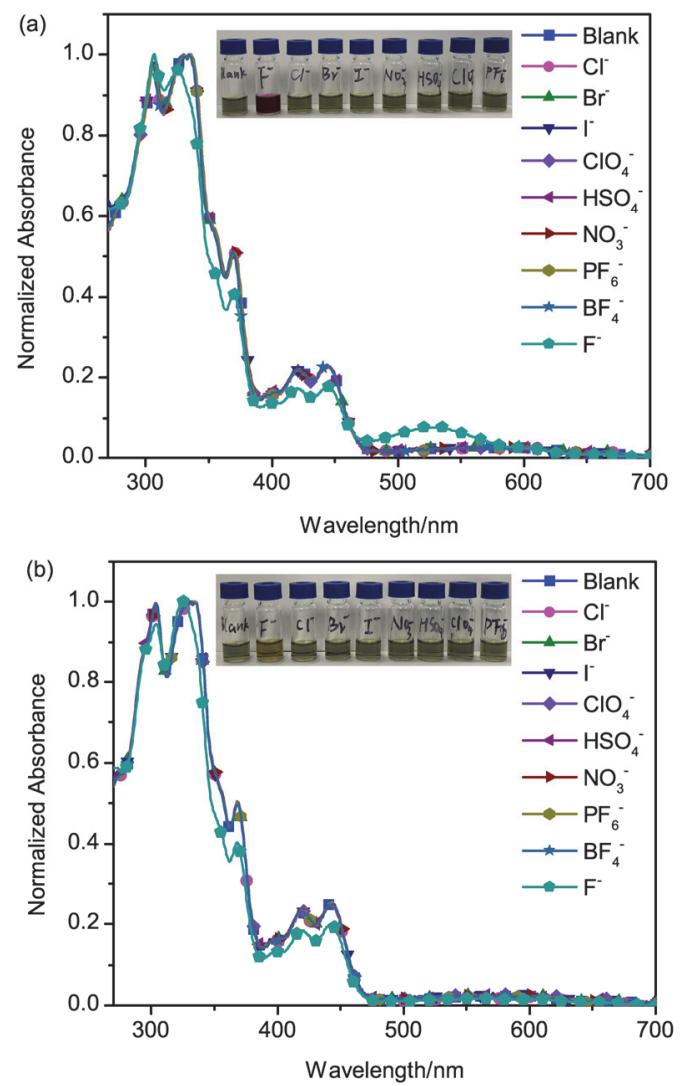

图 7 (a) $1 \mathbf{a}$ 的二氯甲烷溶液中加入不同阴离子的四丁基铵盐 TBAX $\left(n_{\mathrm{TBAX}} / n_{1 \mathrm{a}}=10\right)$ 后的紫外-可见吸收光谱及颜色变化; (b) $1 \mathbf{b}$ 的二氯甲烷 溶液中加入不同阴离子的四丁基铵盐 $\mathrm{TBAX}\left(n_{\mathrm{TBAX}} / n_{\mathbf{1 b}}=10\right)$ 后的紫外可见吸收光谱及颜色变化

Figure 7 (a) UV-Vis absorption spectra and colour change of 1a after addition of tetrabutylammonium salts TBAX of different anions $\left(n_{\mathrm{TBAX}} / n_{1 \mathrm{a}}=10\right)$ in dichloromethane solutions; (b) UV-Vis absorption spectra and colour change of $\mathbf{1 b}$ after addition of tetrabutylammonium salts TBAX of different anions $\left(n_{\mathrm{TBAX}} / n_{\mathbf{1 b}}=10\right)$ in dichloromethane solutions 
具有高度选择性的识别. 另一方面, 氟离子难以与大位 阻 Mes 基保护的 $\mathbf{1 b}$ 中的硼原子发生配位, 吸收光谱在 加入 TBAF 后变化不明显(无新峰产生, 图 7b). 明显的 颜色变化和选择性识别使得 $\mathbf{1 a}$ 有望用作氟离子探针.

\section{3 结论}

我们设计合成了分别含有 $\mathrm{B}-\mathrm{N}$ 键和 $\mathrm{C}=\mathrm{C}$ 键等电 子体的宷基[4]螺烯分子 $\mathbf{1 a} / \mathbf{1 b}$ 和 $\mathbf{2}$, 其中关键合成步骤 分别为亲电硼化反应和铂催化的炔烃环化异构化反应. 实验数据分析和理论计算结果表明 $\mathrm{B}-\mathrm{N}$ 键可以调控共 轭骨架的电子结构, 1a 相对于 $\mathbf{2}$ 的 HOMO-LUMO 能隙 有一定程度的拓宽. 单晶结构解析表明 $1 \mathrm{a}$ 具有典型的 螺烯结构, 晶体中存在 $P$ 和 $M$ 两种对映异构体. $\mathrm{B}-\mathrm{N}$ 键具有部分双键性质, 嗍氮六元环具有较弱的芳香性. 大位阻的 Mes 基使 $\mathbf{1 b}$ 在 TFA 作用下发生和 $\mathbf{2}$ 相似的可 逆的质子化, 而 $1 \mathbf{a}$ 在 TFA 作用下发生脱硼化反应. 此 外, 三配位的硣原子可以进一步和氟离子进行配位从而 改变电子结构, 1a 对氟离子有明显的选择性响应. 该类 螺烯分子有望用于酸致变色和氟离子检测材料. 本工作 丰富了硼氮杂螺烯及全碳螺烯的种类, 对宷基螺烯及薁 基多环芳烃 “自下而上” 的合成及性质研究具有科学意 义.

\section{4 实验部分}

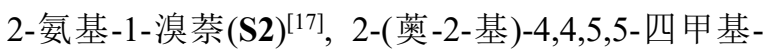
1,3,2-二氧杂硼烷 $(\mathbf{S 3})^{[14 a]}$ 和 1-溴菜-2-基三氟甲磺酸酯 (S6) ${ }^{[18]}$ 根据文献报道方法合成得到.

1-(宷-2-基)菜-2-胺(S4): $200 \mathrm{~mL}$ Schlenk 瓶中依次加 入化合物 S3 (1.02 g, $4 \mathrm{mmol})$, 化合物 S2 (1.07g, 4.8 $\mathrm{mmol}), \mathrm{Pd}(\mathrm{OAc})_{2}(27 \mathrm{mg}, 0.12 \mathrm{mmol}), 2$-双环已基膦2',6'-二甲氧基联苯(Sphos) (99 mg, $0.24 \mathrm{mmol}$ ), $\mathrm{K}_{2} \mathrm{CO}_{3}$ $(1.66 \mathrm{~g}, 12 \mathrm{mmol})$, 抽换氮气三次, 氮气氛围下加入 24 $\mathrm{mL}$ 重蒸四氢呋喃(THF)和 $6 \mathrm{~mL}$ 除氧的水, 搅拌下升温 至 $80{ }^{\circ} \mathrm{C}$ 反应 $10 \mathrm{~h}$. 反应结束后将反应液倒入 $20 \mathrm{~mL}$ 水 中, EtOAc 萃取 $(20 \mathrm{~mL} \times 3)$, 合并有机相, 用饱和氯化钠 溶液洗涤, 无水硫酸钠干燥, 浓缩后柱层析 ( $V$ (石油醚 $(\mathrm{PE})): V(\mathrm{EtOAc})=10: 1)$, 分离得到绿色固体 $656 \mathrm{mg}$, 产率 61\%. m.p. 148.4 149.8 ${ }^{\circ} \mathrm{C} ;{ }^{1} \mathrm{H}$ NMR $(400 \mathrm{MHz}$, Chloroform- $d$ ) $\delta: 8.39$ (d, $J=9.4 \mathrm{~Hz}, 2 \mathrm{H}), 7.76(\mathrm{dd}, J=$ 8.0, $0.8 \mathrm{~Hz}, 1 \mathrm{H}), 7.74(\mathrm{~d}, J=8.8 \mathrm{~Hz}, 1 \mathrm{H}), 7.68 \sim 7.60(\mathrm{~m}$, $2 \mathrm{H}), 7.55(\mathrm{~s}, 2 \mathrm{H}), 7.32 \sim 7.22(\mathrm{~m}, 4 \mathrm{H}), 7.08(\mathrm{~d}, J=8.7 \mathrm{~Hz}$, 1H), 3.94 (s, 2H). ${ }^{13} \mathrm{C}$ NMR (101 MHz, Chloroform-d) $\delta$ : $147.18,141.45,140.75,136.76,135.92,133.49,129.17$, $128.00,127.92,126.44,124.50,123.49,122.29,119.64$, 118.11, 116.10. HRMS (EI) $(m / z): \mathrm{M}^{+}$calcd for $\mathrm{C}_{20} \mathrm{H}_{15} \mathrm{~N}$ 269.1192 , found 269.1199 .

8 - 苯基-7,8-二氢宷 $[1,2-c]$ 菜 $[1,2-e][1,2]$ 氮杂嗍嗪 (1a): $10 \mathrm{~mL}$ 封管中加入化合物 $\mathbf{S 4}(220 \mathrm{mg}, 0.82 \mathrm{mmol})$,
抽换氮气三次, 手套箱内加入 $3 \mathrm{~mL}$ 超干邻二氯苯, 无 水三乙胺 $(165 \mathrm{mg}, 1.63 \mathrm{mmol}), \mathrm{PhBCl}_{2}$ (260 mg, 1.63 $\mathrm{mmol})$, 室温摚拌 $10 \mathrm{~min}$, 然后升温至 $180{ }^{\circ} \mathrm{C}$ 反应 $12 \mathrm{~h}$. 反应体系恢复室温后将反应液倒入 $20 \mathrm{~mL}$ 水中, EtOAc 萃取 $(10 \mathrm{~mL} \times 3)$, 合并有机相, 并用饱和氯化钠溶液洗 涤, 无水硫酸钠干燥, 浓缩后柱层析 $(V(\mathrm{PE}): V(\mathrm{EtOAc})$ $=20 ： 1$ ), 分离得到绿色固体 $230 \mathrm{mg}$, 产率 79\%. m.p. 154.2 155.3 ${ }^{\circ} \mathrm{C}$; ${ }^{1} \mathrm{H}$ NMR (600 MHz, Methylene Chloride- $\left.d_{2}\right) \delta: 9.41(\mathrm{~d}, J=8.4 \mathrm{~Hz}, 1 \mathrm{H}), 8.92(\mathrm{~d}, J=9.0 \mathrm{~Hz}$, $1 \mathrm{H}), 8.73(\mathrm{~s}, 1 \mathrm{H}), 8.63(\mathrm{~d}, J=10.2 \mathrm{~Hz}, 1 \mathrm{H}), 8.03 \sim 7.90$ $(\mathrm{m}, 5 \mathrm{H}), 7.80(\mathrm{td}, J=7.2,1.2 \mathrm{~Hz}, 1 \mathrm{H}), 7.63(\mathrm{td}, J=9.6,0.6$ $\mathrm{Hz}, 1 \mathrm{H}), 7.61 \sim 7.51(\mathrm{~m}, 5 \mathrm{H}), 7.41(\mathrm{t}, J=9.6 \mathrm{~Hz}, 1 \mathrm{H}), 7.28$ $(\mathrm{t}, J=9.6 \mathrm{~Hz}, 1 \mathrm{H}) .{ }^{13} \mathrm{C}$ NMR $(101 \mathrm{MHz}$, Methylene Chloride- $\left.d_{2}\right) \delta: 151.68,146.52,144.46,140.00,136.06,135.82$, $135.59,132.79,132.77,132.42,130.25,130.23,128.98$, $128.47,128.26,127.32,126.17,125.57,123.99,120.77$, 117.68, 115.16. ${ }^{11} \mathrm{~B}$ NMR (128 MHz, Chloroform- $\left.d\right) \delta$ : 36.26. HRMS (DART) $(\mathrm{m} / \mathrm{z}):(\mathrm{M}+\mathrm{H})^{+}$calcd for $\mathrm{C}_{26} \mathrm{H}_{19} \mathrm{~N}^{10} \mathrm{~B} 355.1638$, found 355.1641.

8-(2,4,6-三甲基苯) 基 -7,8-二氢 宷 [1,2-c] 萫 [1,2-e][1,2]氮杂嗍嗪 $(\mathbf{1 b}): 10 \mathrm{~mL}$ 封管中加入化合物 $\mathbf{S 4}$ $(160 \mathrm{mg}, 0.59 \mathrm{mmol})$, 抽换氮气三次, 氮气氛围下加入 3 $\mathrm{mL}$ 超干邻二氯苯, 无水三乙胺 $(120 \mathrm{mg}, 1.19 \mathrm{mmol})$, $\mathrm{BCl}_{3}(1.0 \mathrm{~mol} / \mathrm{L}$ 的对二甲苯溶液, $1.2 \mathrm{~mL}, 1.19 \mathrm{mmol})$, 室温搅拌 $10 \mathrm{~min}$, 然后升温至 $180{ }^{\circ} \mathrm{C}$ 反应 $12 \mathrm{~h}$ 后降至室 温, 加入 $\mathrm{MesMgBr}(1.0 \mathrm{~mol} / \mathrm{L}$ 的 THF 溶液, $2.97 \mathrm{~mL}$, $2.97 \mathrm{mmol}), 60{ }^{\circ} \mathrm{C}$ 反应过夜. 反应结束后用水淬灭反应, EtOAc 萃取 $(10 \mathrm{~mL} \times 3)$, 合并有机相, 并用饱和氯化钠 溶液洗涤, 无水硫酸钠干燥, 浓缩后柱层析 $(V(\mathrm{PE})$ : $V(\mathrm{EtOAc})=20: 1)$, 分离得到绿色固体 $178 \mathrm{mg}$, 产率 75\%. m.p. 196.9 197.7 ${ }^{\circ} \mathrm{C}$; ${ }^{1} \mathrm{H}$ NMR (600 MHz, Methylene Chloride- $\left.d_{2}\right) \delta: 9.45(\mathrm{~d}, J=8.4 \mathrm{~Hz}, 1 \mathrm{H}), 8.75(\mathrm{~s}, 1 \mathrm{H})$, 8.64 (d, $J=10.2 \mathrm{~Hz}, 1 \mathrm{H}), 8.38$ (d, $J=9.0 \mathrm{~Hz}, 1 \mathrm{H}), 8.00$ (d, $J=7.8 \mathrm{~Hz}, 1 \mathrm{H}), 7.98$ (s, 1H), 7.95 (d, $J=8.4 \mathrm{~Hz}, 1 \mathrm{H}), 7.81$ $(\mathrm{td}, J=7.8,1.2 \mathrm{~Hz}, 1 \mathrm{H}), 7.60(\mathrm{t}, J=9.6 \mathrm{~Hz}, 1 \mathrm{H}), 7.57(\mathrm{t}$, $J=7.2 \mathrm{~Hz}, 1 \mathrm{H}), 7.52(\mathrm{~d}, J=9.0 \mathrm{~Hz}, 1 \mathrm{H}), 7.40(\mathrm{t}, J=9.6$ $\mathrm{Hz}, 1 \mathrm{H}), 7.21(\mathrm{t}, J=9.6 \mathrm{~Hz}, 1 \mathrm{H}), 7.03(\mathrm{~s}, 2 \mathrm{H}), 2.43(\mathrm{~s}, 3 \mathrm{H})$, $2.20(\mathrm{~s}, 6 \mathrm{H}) .{ }^{13} \mathrm{C}$ NMR (101 MHz, Methylene Chloride- $\left.d_{2}\right)$ $\delta: 151.18,146.88,144.47,140.33,139.98,137.55,135.97$, $135.51,134.71,132.57,130.18,130.05,130.04,128.98$, $128.96,127.47,127.32,126.38,125.53,125.46,123.92$, 120.93, 117.50, 115.18, 22.38, 21.14. ${ }^{11} \mathrm{~B}$ NMR (128 MHz, Chloroform- $d$ ) $\delta:$ 37.36. HRMS (DART) $(\mathrm{m} / z):(\mathrm{M}+\mathrm{H})^{+}$ calcd for $\mathrm{C}_{29} \mathrm{H}_{25} \mathrm{~N}^{10} \mathrm{~B} 397.2108$, found 397.2111.

1-溴-2-(辛-1-炔-1-基)菜(S7): $25 \mathrm{~mL}$ 反应管中依次 加入化合物 S6 (2.9 g, $8.17 \mathrm{mmol}), \mathrm{CuI}$ (155 mg, 0.82 $\mathrm{mmol}), \mathrm{Pd}\left(\mathrm{PPh}_{3}\right)_{2} \mathrm{Cl}_{2}(286 \mathrm{mg}, 0.41 \mathrm{mmol})$, 抽换氮气三 次, 氮气保护下加入 $20 \mathrm{~mL}$ 无水 $N, N$ 二 二异丙基乙胺 
(DIEPA), $20 \mathrm{~mL}$ 无水 $N, N$-二甲基甲酰胺(DMF)及 1 -辛炔 (2.5 mL, $16.33 \mathrm{mmol})$. 室温搅拌反应 $12 \mathrm{~h}$. 反应结束后 将反应液倒入 $200 \mathrm{~mL}$ 水中, EtOAc 萃取 $(50 \mathrm{~mL} \times 3)$, 合 并有机相, 并用饱和氯化钠溶液洗涤, 无水硫酸钠干燥, 浓缩后以 $\mathrm{PE}$ 为洗脱剂柱层析, 分离得到淡黄色油状物 $2.5 \mathrm{~g}$, 产率 97\%. ${ }^{1} \mathrm{H}$ NMR (400 MHz, Chloroform- $d$ ) $\delta$ : 8.27 (d, $J=8.8 \mathrm{~Hz}, 1 \mathrm{H}), 7.77$ (d, $J=8.0 \mathrm{~Hz}, 1 \mathrm{H}), 7.70$ (d, $J=8.4 \mathrm{~Hz}, 1 \mathrm{H}), 7.58$ (ddd, $J=8.4,6.8,1.2 \mathrm{~Hz}, 1 \mathrm{H})$, $7.52 \sim 7.44(\mathrm{~m}, 2 \mathrm{H}), 2.52(\mathrm{t}, J=6.8 \mathrm{~Hz}, 2 \mathrm{H}), 1.68(\mathrm{p}, J=$ $6.8 \mathrm{~Hz}, 2 \mathrm{H}), 1.61 \sim 1.49(\mathrm{~m}, 2 \mathrm{H}), 1.40 \sim 1.32(\mathrm{~m}, 4 \mathrm{H}), 0.92$ (t, $J=6.8 \mathrm{~Hz}, 3 \mathrm{H}) .{ }^{13} \mathrm{C}$ NMR (101 MHz, Chloroform- $d$ ) $\delta$ : $133.31,132.22,129.35,128.09,127.69,127.27,126.71$, 125.90, 124.19, 96.62, 80.70, 31.39, 28.61, 28.58, 22.61, 19.78, 14.10. HRMS (EI) $(m / z): \mathrm{M}^{+}$calcd for $\mathrm{C}_{18} \mathrm{H}_{19} \mathrm{Br}$ 314.0668 , found 314.0665 .

1-(苸-2-基)-2-(辛-1-炔-1-基)葈(S8)：10 mL 封管中 依次加入化合物 S7 (40 mg, $0.13 \mathrm{mmol})$, 化合物 S3 (38.7 $\mathrm{mg}, 0.15 \mathrm{mmol}), \mathrm{Pd}\left(\mathrm{PPh}_{3}\right)_{4}(7.3 \mathrm{mg}, 0.006 \mathrm{mmol})$ 和 $\mathrm{K}_{2} \mathrm{CO}_{3}(52.7 \mathrm{mg}, 0.38 \mathrm{mmol})$, 抽换氮气三次, 氮气保护 下加入 $2 \mathrm{~mL}$ 重蒸 $\mathrm{THF}$ 和 $0.5 \mathrm{~mL}$ 除氧的水, 搅拌下升温 至 $80{ }^{\circ} \mathrm{C}$ 反应 $10 \mathrm{~h}$. 反应结束后将反应液倒入 $10 \mathrm{~mL}$ 水 中, EtOAc 萃取 $(10 \mathrm{~mL} \times 3)$, 合并有机相, 并用饱和氯化 钠溶液洗涤, 无水硫酸钠干燥, 浓缩后以 $\mathrm{PE}$ 为洗脱剂 柱层析, 分离得到蓝色油状物 $40 \mathrm{mg}$, 产率 $87 \%$. ${ }^{1} \mathrm{H}$ NMR (400 MHz, Chloroform- $d$ ) $\delta: 8.35$ (d, $J=9.6 \mathrm{~Hz}$, 2H), 7.85 (d, $J=8.4 \mathrm{~Hz}, 1 \mathrm{H}), 7.83$ (d, $J=8.2 \mathrm{~Hz}, 1 \mathrm{H}), 7.77$ $(\mathrm{d}, J=8.4 \mathrm{~Hz}, 1 \mathrm{H}), 7.62(\mathrm{~s}, 2 \mathrm{H}), 7.58(\mathrm{t}, J=8.4 \mathrm{~Hz}, 2 \mathrm{H})$, $7.44(\mathrm{td}, J=6.8,1.2 \mathrm{~Hz}, 1 \mathrm{H}), 7.35(\mathrm{td}, J=8.2,6.8,1.2 \mathrm{~Hz}$, $1 \mathrm{H}), 7.19$ (t, $J=9.6 \mathrm{~Hz}, 2 \mathrm{H}), 2.20(\mathrm{t}, J=6.8 \mathrm{~Hz}, 2 \mathrm{H}), 1.31$ $(\mathrm{p}, J=6.8 \mathrm{~Hz}, 2 \mathrm{H}), 1.11 \sim 0.98(\mathrm{~m}, 6 \mathrm{H}), 0.78(\mathrm{t}, J=6.8$ $\mathrm{Hz}, 3 \mathrm{H}) .{ }^{13} \mathrm{C}$ NMR (101 MHz, Chloroform- $d$ ) $\delta: 148.69$, $139.85,138.70,136.65,136.13,132.73,132.12,129.23$, $127.87,127.48,126.89,126.38,125.97,123.04,121.17$, $120.48,93.93,81.24,31.34,28.42,28.31,22.40,19.59$, 14.08. HRMS (EI) $(\mathrm{m} / \mathrm{z}): \mathrm{M}^{+}$calcd for $\mathrm{C}_{28} \mathrm{H}_{26} 362.2024$, found 362.2029 .

8-正己基宷 $[1,2-c]$ 菲(2): $10 \mathrm{~mL}$ 封管中依次加入化 合物 $\mathbf{S 8}$ (40 mg, $0.11 \mathrm{mmol}), \mathrm{PtCl}_{2}(2.9 \mathrm{mg}, 0.01 \mathrm{mmol})$, 抽换氮气三次, 氮气保护下加入 $2 \mathrm{~mL}$ 无水甲苯. 升温 至 $100{ }^{\circ} \mathrm{C}$ 反应 $7 \mathrm{~h}$, 恢复室温用水淬灭反应, EtOAc 萃取 $(10 \mathrm{~mL} \times 3)$, 合并有机相, 并用饱和氯化钠溶液洗涤, 无水硫酸钠干燥, 浓缩后以 $\mathrm{PE}$ 为洗脱剂柱层析, 分离 得到绿色固体 $25 \mathrm{mg}$, 产率 $63 \%$. m.p. 97.3 98.3 ${ }^{\circ} \mathrm{C} ;{ }^{1} \mathrm{H}$ NMR (600 MHz, Chloroform- $d$ ) $\delta: 9.45$ (d, $J=8.4 \mathrm{~Hz}$, $1 \mathrm{H}), 8.87(\mathrm{~d}, J=9.6 \mathrm{~Hz}, 1 \mathrm{H}), 8.75(\mathrm{~s}, 1 \mathrm{H}), 8.40(\mathrm{~d}, J=$ $10.2 \mathrm{~Hz}, 1 \mathrm{H}), 8.03$ (dd, $J=7.2,0.6 \mathrm{~Hz}, 1 \mathrm{H}), 7.96 \sim 7.89$ (m, 2H), $7.81(\mathrm{td}, J=7.2,1.2 \mathrm{~Hz}, 1 \mathrm{H}), 7.75(\mathrm{~s}, 1 \mathrm{H}), 7.65$ (td, $J=7.2,1.2 \mathrm{~Hz}, 1 \mathrm{H}), 7.49$ (dd, $J=9.6,0.6 \mathrm{~Hz}, 1 \mathrm{H})$, $7.30(\mathrm{dd}, J=9.6,0.6 \mathrm{~Hz}, 1 \mathrm{H}), 7.13(\mathrm{dd}, J=10.2,9.6 \mathrm{~Hz}$,
1H), 3.55 (t, $J=7.8 \mathrm{~Hz}, 2 \mathrm{H}), 2.03$ (p, $J=7.8 \mathrm{~Hz}, 2 \mathrm{H}), 1.62$ $(\mathrm{p}, J=7.8 \mathrm{~Hz}, 2 \mathrm{H}), 1.48 \sim 1.35(\mathrm{~m}, 4 \mathrm{H}), 0.95(\mathrm{t}, J=7.2$ $\mathrm{Hz}, 3 \mathrm{H}) .{ }^{13} \mathrm{C}$ NMR (101 MHz, Chloroform-d) $\delta: 140.99$, $140.28,138.78,138.24,136.75,135.44,133.34,132.89$, $132.56,131.62,128.72,128.03,127.35,127.30,126.62$, $126.47,125.30,124.90,127.88,123.44,122.23,118.04$, 35.91, 31.81, 29.71, 29.07, 22.69, 14.13. HRMS (EI) $(\mathrm{m} / \mathrm{z})$ : $\mathrm{M}^{+}$calcd for $\mathrm{C}_{28} \mathrm{H}_{26} 362.2025$, found 362.2029.

\section{References}

[1] (a) Clar, E. Polycyclic Hydrocarbons, Academic Press, London 1964. (b) Anthony, J. E. Chem. Rev. 2006, 106, 5028. (c) Wu, J.; Pisula, W.; Müllen, K. Chem. Rev. 2007, 107, 718. (d) Wang, X.-Y.; Yao, X.; Müllen, K. Sci. China Chem. 2019, 62, 1099.

[2] (a) Stępień, M.; Gońka, E.; Żyła, M.; Sprutta, N. Chem. Rev. 2017, 117, 3479. (b) Hirai, M.; Tanaka, N.; Sakai, M.; Yamaguchi, S Chem. Rev. 2019, 119, 8291. (c) Lv, M.; Zhou, R.-M.; Lv, K.; Wei, Z.-X. Acta Chim. Sinica 2021, 79, 284 (in Chinese). (吕敏, 周瑞敏, 吕琨, 魏志祥, 化学学报, 2021, 79, 284). (d) Li, L.-Y.; Zheng, W.; Li, C.-H. Acta Chim. Sinica 2021, 79, 81 (in Chinese); (李凌燕, 郑 玮, 李承辉, 化学学报, 2021, 79, 81). (e) Hu, X.-M.; Zhong, C.-X.; Li, X.-Y.; Jia, X.; Wei, Y.; Xie, L.-H. Acta Chim. Sinica 2021, 79 , 953 (in Chinese). (胡金明, 钟春晓, 李晓燕, 贾雄, 魏颖, 解令海, 化学学报, 2021, 79, 953).

[3] (a) Bosdet, M. J. D.; Piers, W. E. Can. J. Chem. 2009, 87, 8. (b) Jaska, C. A.; Emslie, D. J. H.; Bosdet, M. J. D.; Piers, W. E.; Sorensen, T. S.; Parvez, M. J. Am. Chem. Soc. 2006, 128, 10885. (c) Wang, X.-Y.; Zhuang, F.-D.; Wang, R.-B.; Wang, X.-C.; Cao, X.-Y.; Wang, J.-Y.; Pei, J. J. Am. Chem. Soc. 2014, 136, 3764. (d) Dou, C.; Ding, Z.; Zhang, Z.; Xie, Z.; Liu, J.; Wang, L. Angew. Chem. Int. Ed. 2015, 54, 3648. (e) Zhuang, F.-D.; Sun, Z.-H.; Yao, Z.-F.; Chen, Q.-R.; Huang, Z.; Yang, J.-H.; Wang, J.-Y.; Pei, J. Angew. Chem. Int. Ed. 2019, 58, 10708. (f) Wang, X.; Zhang, F.; Schellhammer, K. S.; Machata, P.; Ortmann, F.; Cuniberti, G.; Fu, Y.; Hunger, J.; Tang, R.; Popov, A. A.; Berger, R.; Müllen, K.; Feng, X. J. Am. Chem. Soc. 2016, 138, 11606. (g) Cao, Y.; Zhu, C.; Barlog, M.; Barker, K. P.; Ji, X.; Kalin, A. J.; Al-Hashimi, M.; Fang, L. J. Org. Chem. 2021, 86, 2100. (h) Abengózar, A.; García-García, P.; Sucunza, D.; Pérez-Redondo, A.; Vaquero, J. J. Chem. Commun. 2018, 54, 2467.

[4] (a) Wang, S.; Yang, D.-T.; Lu, J.; Shimogawa, H.; Gong, S.; Wang, X.; Mellerup, S. K.; Wakamiya, A.; Chang, Y.-L.; Yang, C.; Lu, Z.-H. Angew. Chem. Int. Ed. 2015, 54, 15074. (b) Hashimoto, S.; Ikuta, T.; Shiren, K.; Nakatsuka, S.; Ni, J.; Nakamura, M.; Hatakeyama, T. Chem. Mater. 2014, 26, 6265. (c) Qiang, P.; Sun, Z.; Wan, M.; Wang, X.; Thiruvengadam, P.; Bingi, C.; Wei, W.; Zhu, W.; Wu, D.; Zhang, F. Org. Lett. 2019, 21, 4575.

[5] Crossley, D. L.; Cade, I. A.; Clark, E. R.; Escande, A.; Humphries, M. J.; King, S. M.; Vitorica-Yrezabal, I.; Ingleson, M. J.; Turner, M. L. Chem. Sci. 2015, 6, 5144.

[6] Huang, H.; Zhou, Y.; Wang, M.; Zhang, J.; Cao, X.; Wang, S.; Cao, D.; Cui, C. Angew. Chem. Int. Ed. 2019, 58, 10132.

[7] (a) Martin, R. H. Angew. Chem. Int. Ed. Engl. 1974, 13, 649. (b) Shen, Y.; Chen, C.-F. Chem. Rev. 2012, 112, 1463. (c) Gingras, M.; Felix, G.; Peresutti, R. Chem. Soc. Rev. 2013, 42, 1007. (d) Gingras, M. Chem. Soc. Rev. 2013, 42, 1051. (e) Gingras, M. Chem. Soc. Rev. 2013, 42, 968 .

[8] (a) Yamamoto, K.; Shimizu, T.; Igawa, K.; Tomooka, K.; Hirai, G.; Suemune, H.; Usui, K. Sci. Rep. 2016, 6, 36211. (b) Nakamura, K.; Furumi, S.; Takeuchi, M.; Shibuya, T.; Tanaka, K. J. Am. Chem. Soc. 2014, 136, 5555. (c) Isla, H.; Saleh, N.; Ou-Yang, J.-K.; Dhbaibi, K.; Jean, M.; Dziurka, M.; Favereau, L.; Vanthuyne, N.; Toupet, L.; Jamoussi, B.; Srebro-Hooper, M.; Crassous, J. J. Org. Chem. 2019, 84, 5383. (d) Schulte, T. R.; Holstein, J. J.; Clever, G. H. Angew. Chem. Int. Ed. 2019, 58, 5562. (e) Gicquel, M.; Zhang, Y.; Aillard, P.; Retailleau, P.; Voituriez, A.; Marinetti, A. Angew. Chem., Int. Ed. 2015, 54, 5470. (f) Li, M.; Lin, W.-B.; Fang, L.; Chen, C.-F. Acta Chim. Sinica 2017, 75, 1150 (in Chinese). (李猛, 林伟涁, 房蕾, 陈 传峰, 化学学报, 2017, 75, 1150). (g) Chen, X.-Y.; Li, J.-K.; Wang, X.-Y. Chin. J. Org. Chem. 2021, 41, 4105 (in Chinese). (陈星宇, 李 继坤, 王小野, 有机化学, 2021, 41, 4105). 
[9] Cahn, R. S.; Ingold, C.; Prelog, V. Angew. Chem. Int. Ed. Engl. 1966, $5,385$.

[10] Martin, R. H.; Marchant, M. J. Tetrahedron 1974, 30, 347

[11] (a) Wheland, G. W.; Mann, D. E. J. Chem. Phys. 1949, 17, 264. (b) Anderson, A. G.; Steckler, B. M. J. Am. Chem. Soc. 1959, 81, 4941.

[12] Michl, J.; Thulstrup, E. W. Tetrahedron 1976, 32, 205.

[13] Beer, M.; Longuet-Higgins, H. C. J. Chem. Phys. 1955, 23, 1390.

[14] (a) Xin, H.; Li, J.; Yang, X.; Gao, X. J. Org. Chem. 2020, 85, 70. (b) Pigulski, B.; Shoyama, K.; Würthner, F. Angew. Chem. Int. Ed. 2020, 59, 15908. (c) Xin, H.; Li, J.; Lu, R.-Q.; Gao, X.; Swager, T. M. J. Am. Chem. Soc. 2020, 142, 13598. (d) Sasaki, Y.; Takase, M.; Okujima, T; Mori, S; Uno, H. Org. Lett. 2019, 21, 1900. (e) Smits, E. C. P.; Setayesh, S.; Anthopoulos, T. D.; Buechel, M.; Nijssen, W.; Coehoorn, R.; Blom, P. W. M.; de Boer, B.; de Leeuw, D. M. Adv. Mater. 2007, 19, 734. (f) Yamaguchi, Y.; Maruya, Y.; Katagiri, H.; Nakayama, K.-i.; Ohba, Y. Org. Lett. 2012, 14, 2316. (g) Yamaguchi, Y.; Ogawa, K.; Nakayama, K.-i.; Ohba, Y.; Katagiri, H. J. Am. Chem. Soc. 2013, 135, 19095. (h) Yamaguchi, Y.; Takubo, M.; Ogawa, K.; Nakayama, K.-i.; Koganezawa, T.; Katagiri, H. J. Am. Chem. Soc. 2016, 138, 11335. (i) Xin, H.; Ge, C.; Yang, X.; Gao, H.; Yang, X.; Gao, X. Chem. Sci. 2016, 7, 6701. (j) Xin, H.; Ge, C.; Jiao, X.; Yang, X.; Rundel, K.; McNeill, C. R.; Gao, X. Angew. Chem. Int. Ed. 2018, 57, 1322.

[15] (a) Zhu, C.; Shoyama, K.; Würthner, F. Angew. Chem. Int. Ed. 2020, 59, 21505. (b) Zhang, X.-S.; Huang, Y.-Y.; Zhang, J.; Meng, W.; Peng, Q.; Kong, R.; Xiao, Z.; Liu, J.; Huang, M.; Yi, Y.; Chen, L.; Fan, Q.; Lin, G.; Liu, Z.; Zhang, G.; Jiang, L.; Zhang, D. Angew. Chem. Int. Ed. 2020, 59, 3529. (c) Ogawa, N.; Yamaoka, Y.; Takikawa, H.; Yamada, K.-i.; Takasu, K. J. Am. Chem. Soc. 2020, 142, 13322. (d) Konishi, A.; Horii, K.; Shiomi, D.; Sato, K.; Takui, T.; Yasuda, M. J. Am. Chem. Soc. 2019, 141, 10165. (e) Liu, J.; Mishra, S.; Pignedoli, C. A.; Passerone, D.; Urgel, J. I.; Fabrizio, A.; Lohr, T. G.; Ma, J.; Komber, H.; Baumgarten, M.; Corminboeuf, C.; Berger, R.; Ruffieux, P.; Müllen, K.; Fasel, R.; Feng, X. J. Am. Chem. Soc. 2019, 141, 12011. (f) Yang, X.; Rominger, F.; Mastalerz, M. Angew. Chem. Int. Ed. 2019, 58, 17577. (g) Zhu, C.; Shoyama, K.; Würthner, F. Angew. Chem. Int. Ed. 2020, 59, 21505. (h) Yamamoto, K. I. Y.; Tohnai, N.; Kakiuchi, F.; Aso, Y. Sci. Rep. 2018, 8,17663 .

[16] (a) Uehara, K.; Mei, P.; Murayama, T.; Tani, F.; Hayashi, H.; Suzuki, M.; Aratani, N.; Yamada, H. Eur. J. Org. Chem. 2018, 4508. (b) Yamamoto, K.; Okazumi, M. Suemune, H.; Usui, K. Org. Lett. 2013, 15, 1806. (c) Han, Y.; Xue, Z.; Li, G.; Gu, Y.; Ni, Y.; Dong, S.; Chi, C. Angew. Chem. Int. Ed. 2020, 59, 9026. (d) Ma, J.; Fu, Y.; Dmitrieva, E.; Liu, F.; Komber, H.; Hennersdorf, F.; Popov, A. A.; Weigand, J. J.; Liu, J.; Feng, X. Angew. Chem. Int. Ed. 2020, 59, 5637.

[17] Ortgies, S.; Breder, A. Org. Lett. 2015, 17, 2748.

[18] Weimar, M.; Correa da Costa, R.; Lee, F.-H.; Fuchter, M. J. Org. Lett. 2013, 15, 1706

[19] Niu, W.; Ma, J.; Soltani, P.; Zheng, W.; Liu, F.; Popov, A. A.; Weigand, J. J.; Komber, H.; Poliani, E.; Casiraghi, C.; Droste, J.; Hansen, M. R.; Osella, S.; Beljonne, D.; Bonn, M.; Wang, H. I.; Feng, X.; Liu, J.; Mai, Y. J. Am. Chem. Soc. 2020, 142, 18293.

[20] Fürstner, A.; Mamane, V. J. Org. Chem. 2002, 67, 6264.

[21] Abbey, E. R.; Zakharov, L. N.; Liu, S.-Y. J. Am. Chem. Soc. 2008, 130,7250
[22] (a) Chen, Y.; Chen, W.; Qiao, Y.; Lu, X.; Zhou, G. Angew. Chem. Int. Ed. 2020, 59, 7122. (b) Zhuang, F. D.; Yang, J. H.; Sun, Z. H.; Zhang, P. F.; Chen, Q. R.; Wang, J. Y.; Pei, J. Chin. J. Chem. 2021 39, 909. (c) Campbell, P. G.; Marwitz, A. J. V.; Liu, S.-Y. Angew. Chem. Int. Ed. 2012, 51, 6074. (d) Sun, Z.; Yi, C.; Liang, Q.; Bingi, C.; Zhu, W.; Qiang, P.; Wu, D.; Zhang, F. Org. Lett. 2020, 22, 209. (e) Nakatsuka, S.; Yasuda, N.; Hatakeyama, T. J. Am. Chem. Soc. 2018, 140, 13562. (f) Hatakeyama, T.; Hashimoto, S.; Oba, T.; Nakamura, M. J. Am. Chem. Soc. 2012, 134, 19600. (g) Pati, P. B.; Jin, E.; Kim, Y.; Kim, Y.; Mun, J.; Kim, S. J.; Kang, S. J.; Choe, W.; Lee, G.; Shin, H.-J.; Park, Y. S. Angew. Chem. Int. Ed. 2020, 59, 14891.

[23] Biet, T.; Fihey, A.; Cauchy, T.; Vanthuyne, N.; Roussel, C.; Crassous, J.; Avarvari, N. Chem. Eur. J. 2013, 19, 13160.

[24] (a) Ravat, P. Chem. Eur J. 2021, 27, 3957. (b) Liu, B-K. Zhang, Y.-L.; Chen, Y.; Liu, X.-G.; Zhang, L. Chin. J. Org. Chem. 2020, 40, 2879 (in Chinese). (刘秉康, 张艳丽, 陈瑜, 刘旭光, 张否, 有机 化学, 2020, 40, 2879).

[25] Xin, H.; Gao, X. ChemPlusChem 2017, 82, 945.

[26] (a) Murai, M.; Iba, S.; Ota, H.; Takai, K. Org. Lett. 2017, 19, 5585. (b) Hou, B.; Li, J.; Xin, H.-S.; Yang, X.-D.; Gao, H.-L.; Peng, P.-Z.; Gao, X.-K. Acta Chim. Sinica 2020, 78, 788 (in Chinese). (侯斌，李 晶，辛涵申，杨笑迪，高洪否，彭培珍，高希珂，化学学报，2020, $78,788)$.

[27] (a) Ju, C. W.; Li, B.; Li, L.; Yan, W.; Cui, C.; Ma, X.; Zhao, D. J. Am. Chem. Soc. 2021, 143, 5903. (b) Xia, Y.; Zhang, M.; Ren, S.; Song, J.; Ye, J.; Humphrey, M. G.; Zheng, C.; Wang, K.; Zhang, X. Org. Lett. 2020, 22, 7942. (c) Urban, M.; Durka, K.; Jankowski, P.; Serwatowski, J.; Lulinski, S. J. Org. Chem. 2017, 82, 8234.

[28] Murfin, L. C.; Weber, M.; Park, S. J.; Kim, W. T.; Lopez-Alled, C. M.; McMullin, C. L.; Pradaux-Caggiano, F.; Lyall, C. L.; Kociok-Köhn, G.; Wenk, J.; Bull, S. D.; Yoon, J.; Kim, H. M.; James, T. D.; Lewis, S. E. J. Am. Chem. Soc. 2019, 141, 19389.

[29] Brown, A. R.; Jarrett, C. P.; de Leeuw, D. M.; Matters, M. Synth. Met. 1997, 88, 37.

[30] (a) Amir, E.; Amir, R. J.; Campos, L. M.; Hawker, C. J. J. Am. Chem. Soc. 2011, 133, 10046. (b) Murai, M.; Amir, E.; Amir, R. J.; Hawker, C. J. Chem. Sci. 2012, 3, 2721. (c) Amir, E.; Murai, M.; Amir, R. J.; Cowart, J. S.; Chabinyc, M. L.; Hawker, C. J. Chem. Sci. 2014, 5, 4483. (d) Murai, M.; Ku, S.-Y.; Treat, N. D.; Robb, M J.; Chabinyc, M. L.; Hawker, C. J. Chem. Sci. 2014, 5, 3753. (e) Tsurui, K.; Murai, M.; Ku, S.-Y.; Hawker, C. J.; Robb, M. J. Adv. Funct. Mater. 2014, 24, 7338. (f) Peng, P.-Z.; Li, J.; Hou, B.; Xin, H.-S.; Cheng, T.-Y.; Gao, X.-K. Chin. J. Org. Chem. 2020, 40, 3916 (in Chinese). (彭培珍，李晶，侯斌，辛涵申，程探宇，高希珂，有 机化学, 2020, 40, 3916).

[31] (a) Matsuo, K.; Saito, S.; Yamaguchi, S. J. Am. Chem. Soc. 2014, 136, 12580. (b) Schickedanz, K.; Trageser, T.; Bolte, M.; Lerner, H.-W.; Wagner, M. Chem. Commun. 2015, 51, 15808. (c) Miyamoto, F.; Nakatsuka, S.; Yamada, K.; Nakayama, K.-i.; Hatakeyama, T. Org. Lett. 2015, 17, 6158. (d) Schickedanz, K.; Radtke, J.; Bolte, M.; Lerner, H.-W.; Wagner, M. J. Am. Chem. Soc. 2017, 139, 2842. (e) Iida, A.; Yamaguchi, S. J. Am. Chem. Soc. 2011, 133, 6952.

[32] Wade, C. R.; Broomsgrove, A. E. J.; Aldridge, S.; Gabbaï, F. P. Chem. Rev. 2010, 110, 3958. 